\title{
Relaxed Composite Implicit Iteration Process for Common Fixed Points of a Finite Family of Strictly Pseudocontractive Mappings
}

\author{
L. C. Ceng, ${ }^{1}$ David S. Shyu, ${ }^{2}$ and J. C. Yao ${ }^{3}$ \\ ${ }^{1}$ Department of Mathematics, Shanghai Normal University, Shanghai 200234, China \\ ${ }^{2}$ Department of Finance, National Sun Yat-Sen University, Kaohsiung 80424, Taiwan \\ ${ }^{3}$ Department of Applied Mathematics, National Sun Yat-Sen University, Kaohsiung 804, Taiwan
}

Correspondence should be addressed to J. C. Yao, yaojc@math.nsysu.edu.tw

Received 26 November 2008; Accepted 28 May 2009

Recommended by Lai-Jiu Lin

We propose a relaxed composite implicit iteration process for finding approximate common fixed points of a finite family of strictly pseudocontractive mappings in Banach spaces. Several convergence results for this process are established.

Copyright (C) 2009 L. C. Ceng et al. This is an open access article distributed under the Creative Commons Attribution License, which permits unrestricted use, distribution, and reproduction in any medium, provided the original work is properly cited.

\section{Introduction and Preliminaries}

Let $E$ be a real Banach space, and let $E^{*}$ be its dual space. Denote by $J$ the normalized duality mapping from $E$ into $2^{E^{*}}$ defined by

$$
J(x)=\left\{\varphi \in E^{*}:\langle x, \varphi\rangle=\|x\|^{2}=\|\varphi\|^{2}\right\}, \quad \forall x \in E,
$$

where $\langle\cdot, \cdot\rangle$ is the generalized duality pairing between $E$ and $E^{*}$. If $E$ is smooth, then $J$ is single valued and continuous from the norm topology of $E$ to the weak* topology of $E^{*}$.

A mapping $T$ with domain $D(T)$ and range $R(T)$ in $E$ is called $\lambda$-strictly pseudocontractive in the terminology of Browder and Petryshyn [1], if there exists a constant $\lambda>0$ such that

$$
\langle T x-T y, j(x-y)\rangle \leq\|x-y\|^{2}-\lambda\|x-y-(T x-T y)\|^{2}
$$


for all $x, y \in D(T)$ and all $j(x-y) \in J(x-y)$. Without loss of generality, we may assume $\lambda \in(0,1)$. If $I$ denotes the identity operator, then (1.2) can be written in the form

$$
\langle(I-T) x-(I-T) y, j(x-y)\rangle \geq \lambda\|(I-T) x-(I-T) y\|^{2}
$$

for all $x, y \in D(T)$ and all $j(x-y) \in J(x-y)$. In (1.2) and (1.3), the positive number $\lambda>0$ is said to be a strictly pseudocontractive constant.

The class of strictly pseudocontractive mappings has been studied by several authors (see, e.g., [1-10]). It is shown in [4] that a strictly pseudocontractive map is L-Lipschitzian (i.e., $\|T x-T y\| \leq L\|x-y\|, \forall x, y \in D(T)$ for some $L>0$ ). Indeed, it follows immediately from (1.3) that

$$
\|x-y\| \geq \lambda\|(I-T) x-(I-T) y\| \geq \lambda(\|T x-T y\|-\|x-y\|)
$$

and hence $\|T x-T y\| \leq L\|x-y\|, \forall x, y \in D(T)$ where $L=1+1 / \lambda$. It is clear that in Hilbert spaces the important class of nonexpansive mappings (mappings $T$ for which $\|T x-T y\| \leq$ $\|x-y\|, \forall x, y \in D(T))$ is a subclass of the class of strictly pseudocontractive maps.

Let $K$ be a nonempty convex subset of $E$, and let $\left\{T_{i}\right\}_{i=1}^{N}$ be a finite family of nonexpansive self-maps of $K$. In [11], $\mathrm{Xu}$ and Ori introduced the following implicit iteration process; for any initial $x_{0} \in K$ and $\left\{\alpha_{n}\right\}_{n=1}^{\infty} \subset(0,1)$, the sequence $\left\{x_{n}\right\}_{n=1}^{\infty}$ is generated as follows:

$$
\begin{aligned}
x_{1} & =\alpha_{1} x_{0}+\left(1-\alpha_{1}\right) T_{1} x_{1}, \\
x_{2} & =\alpha_{2} x_{1}+\left(1-\alpha_{2}\right) T_{2} x_{2}, \\
& \vdots \\
x_{N} & =\alpha_{N} x_{N-1}+\left(1-\alpha_{N}\right) T_{N} x_{N}, \\
x_{N+1} & =\alpha_{N+1} x_{N}+\left(1-\alpha_{N+1}\right) T_{1} x_{N+1},
\end{aligned}
$$

The scheme is expressed in a compact form as

$$
x_{n}=\alpha_{n} x_{n-1}+\left(1-\alpha_{n}\right) T_{n} x_{n}, \quad n \geq 1,
$$

where $T_{n}=T_{n \text { mod } N}$. Moreover, they proved the following convergence theorem in a Hilbert space.

Theorem 1.1 (see [11]). Let $H$ be a Hilbert space, and let $K$ be a nonempty closed convex subset of $H$. Let $\left\{T_{i}\right\}_{i=1}^{N}$ be $N$ nonexpansive self-maps of $K$ such that $C=\bigcap_{i=1}^{N} F\left(T_{i}\right) \neq \varnothing$ where $F\left(T_{i}\right)=\{x \in$ $\left.K: T_{i} x=x\right\}$. Let $x_{0} \in K$, and let $\left\{\alpha_{n}\right\}_{n=1}^{\infty}$ be a sequence in $(0,1)$, such that $\lim _{n \rightarrow \infty} \alpha_{n}=0$. Then the sequence $\left\{x_{n}\right\}$ defined implicitly by (1.6) converges weakly to a common fixed point of the mappings $\left\{T_{i}\right\}_{i=1}^{N}$. 
Subsequently, Osilike [12] extended their results from nonexpansive mappings to strictly pseudocontractive mappings and derived the following convergence theorems in Hilbert and Banach spaces.

Theorem 1.2 (see [12]). Let $H$ be a real Hilbert space, and let $K$ be a nonempty closed convex subset of $H$. Let $\left\{T_{i}\right\}_{i=1}^{N}$ be $N$ strictly pseudocontractive self-maps of $K$ such that $C=\bigcap_{i=1}^{N} F\left(T_{i}\right) \neq \varnothing$, where $F\left(T_{i}\right)=\left\{x \in K: T_{i} x=x\right\}$. Let $x_{0} \in K$, and let $\left\{\alpha_{n}\right\}_{n=1}^{\infty}$ be a sequence in $(0,1)$ such that $\lim _{n \rightarrow \infty} \alpha_{n}=0$. Then the sequence $\left\{x_{n}\right\}_{n=1}^{\infty}$ defined by (1.6) converges weakly to a common fixed point of the mappings $\left\{T_{i}\right\}_{i=1}^{N}$.

Theorem 1.3 (see [12]). Let $E$ be a real Banach space, and let $K$ be a nonempty closed convex subset of $E$. Let $\left\{T_{i}\right\}_{i=1}^{N}$ be $N$ strictly pseudocontractive self-maps of $K$ such that $C=\bigcap_{i=1}^{N} F\left(T_{i}\right) \neq \varnothing$, where $F\left(T_{i}\right)=\left\{x \in K: T_{i} x=x\right\}$, and let $\left\{\alpha_{n}\right\}_{n=1}^{\infty}$ be a real sequence satisfying the conditions:

(i) $0<\alpha_{n}<1$;

(ii) $\sum_{n=1}^{\infty}\left(1-\alpha_{n}\right)=+\infty$;

(iii) $\sum_{n=1}^{\infty}\left(1-\alpha_{n}\right)^{2}<+\infty$.

Let $x_{0} \in K$, and let $\left\{x_{n}\right\}_{n=1}^{\infty}$ be defined by (1.6). Then

(i) $\lim _{n \rightarrow \infty}\left\|x_{n}-p\right\|$ exists for all $p \in F$;

(ii) $\liminf _{n \rightarrow \infty}\left\|x_{n}-T_{n} x_{n}\right\|=0$.

Let $K$ be a nonempty closed convex subset of a real Banach space $E$. Very recently, $\mathrm{Su}$ and $\mathrm{Li}$ [13] introduced a new implicit iteration process for $N$ strictly pseudocontractive self-maps $\left\{T_{i}\right\}_{i=1}^{N}$ of $K$ :

$$
\begin{gathered}
x_{n}=\alpha_{n} x_{n-1}+\left(1-\alpha_{n}\right) T_{n} y_{n}, \\
y_{n}=\beta_{n} x_{n-1}+\left(1-\beta_{n}\right) T_{n} x_{n}, \quad n=1,2, \ldots,
\end{gathered}
$$

that is,

$$
x_{n}=\alpha_{n} x_{n-1}+\left(1-\alpha_{n}\right) T_{n}\left(\beta_{n} x_{n-1}+\left(1-\beta_{n}\right) T_{n} x_{n}\right), \quad n \geq 1,
$$

where $T_{n}=T_{n \bmod N}$ and $\left\{\alpha_{n}\right\},\left\{\beta_{n}\right\} \subset[0,1]$. First, they established the following convergence theorem.

Theorem 1.4 ([13, Theorem 2.1]). Let $E$ be a real Banach space, and let $K$ be a nonempty closed convex subset of $E$. Let $\left\{T_{i}\right\}_{i=1}^{N}$ be $N$ strictly pseudocontractive self-maps of $K$ such that $C=\bigcap_{i=1}^{N} F\left(T_{i}\right) \neq \varnothing$, where $F\left(T_{i}\right)=\left\{x \in K: T_{i} x=x\right\}$, and let $\left\{\alpha_{n}\right\}_{n=1}^{\infty},\left\{\beta_{n}\right\}_{n=1}^{\infty} \subset[0,1]$ be two real sequences satisfying the conditions:

(i) $\sum_{n=1}^{\infty}\left(1-\alpha_{n}\right)=+\infty$;

(ii) $\sum_{n=1}^{\infty}\left(1-\alpha_{n}\right)^{2}<+\infty$;

(iii) $\sum_{n=1}^{\infty}\left(1-\beta_{n}\right)<+\infty$;

(iv) $\left(1-\alpha_{n}\right)\left(1-\beta_{n}\right) L^{2}<1, \forall n \geq 1$, where $L \geq 1$ is common Lipschitz constant of $\left\{T_{i}\right\}_{i=1}^{N}$. 
For $x_{0} \in K$, let $\left\{x_{n}\right\}_{n=1}^{\infty}$ be defined by (1.8). Then

(i) $\lim _{n \rightarrow \infty}\left\|x_{n}-p\right\|$ exists for all $p \in C$;

(ii) $\liminf _{n \rightarrow \infty}\left\|x_{n}-T_{n} x_{n}\right\|=0$.

Second, they derived the following result by using Theorem 1.4.

Theorem 1.5 ([13, Theorem 2.2]). Let $K$ be a nonempty closed convex subset of a real Banach space $E$, let $T$ be a semicompact strictly pseudocontractive self-map of $K$ such that $F(T) \neq \varnothing$, where $F(T)=\{x \in K: T x=x\}$, and let $\left\{\alpha_{n}\right\} \subset[0,1]$ be a real sequence satisfying the conditions:

(i) $\sum_{n=1}^{\infty}\left(1-\alpha_{n}\right)=+\infty$;

(ii) $\sum_{n=1}^{\infty}\left(1-\alpha_{n}\right)^{2}<+\infty$.

Then for $x_{0} \in K$, the sequence $\left\{x_{n}\right\}$ defined by Mann iterative process,

$$
x_{n}=\alpha_{n} x_{n-1}+\left(1-\alpha_{n}\right) T x_{n-1}, \quad n \geq 1,
$$

converges strongly to a fixed point of $T$.

On the other hand, Zeng and Yao [14] introduced a new implicit iteration scheme with perturbed mapping for approximation of common fixed points of a finite family of nonexpansive self-maps of a real Hilbert space $H$ and established some convergence theorems for this implicit iteration scheme. To be more specific, let $\left\{T_{i}\right\}_{i=1}^{N}$ be a finite family of nonexpansive self-maps of $H$, and let $F: H \rightarrow H$ be a mapping such that for some constants $\kappa, \eta>0 ; F$ is a $\kappa$-Lipschitz and $\eta$-strongly monotone mapping. Let $\left\{\alpha_{n}\right\}_{n=1}^{\infty} \subset(0,1)$ and $\left\{\lambda_{n}\right\}_{n=1}^{\infty} \subset[0,1)$ and take a fixed number $\mu \in\left(0,2 \eta / \kappa^{2}\right)$. The authors proposed the following implicit iteration process with perturbed mapping $F$.

For an arbitrary initial point $x_{0} \in H$, the sequence $\left\{x_{n}\right\}_{n=1}^{\infty}$ is generated as follows:

$$
\begin{aligned}
x_{1} & =\alpha_{1} x_{0}+\left(1-\alpha_{1}\right)\left[T_{1} x_{1}-\lambda_{1} \mu F\left(T_{1} x_{1}\right)\right], \\
x_{2} & =\alpha_{2} x_{1}+\left(1-\alpha_{2}\right)\left[T_{2} x_{2}-\lambda_{2} \mu F\left(T_{2} x_{2}\right)\right], \\
& \vdots \\
x_{N} & =\alpha_{N} x_{N-1}+\left(1-\alpha_{N}\right)\left[T_{N} x_{N}-\lambda_{N} \mu F\left(T_{N} x_{N}\right)\right], \\
x_{N+1} & =\alpha_{N+1} x_{N}+\left(1-\alpha_{N+1}\right)\left[T_{1} x_{N+1}-\lambda_{N+1} \mu F\left(T_{1} x_{N+1}\right)\right],
\end{aligned}
$$

The scheme is expressed in a compact form as

$$
x_{n}=\alpha_{n} x_{n-1}+\left(1-\alpha_{n}\right)\left[T_{n} x_{n}-\lambda_{n} \mu F\left(T_{n} x_{n}\right)\right], \quad n \geq 1 .
$$

It is clear that if $\lambda_{n} \equiv 0$, then the implicit iteration scheme (1.11) with perturbed mapping reduces to the implicit iteration process (1.6). 
Theorem 1.6 ([14, Theorem 2.1]). Let $H$ be a real Hilbert space, and let $F: H \rightarrow H$ be a mapping such that for some constants $\kappa, \eta>0$; $F$ is $\kappa$-Lipschitz and $\eta$-strongly monotone. Let $\left\{T_{i}\right\}_{i=1}^{N}$ be $N$ nonexpansive self-maps of $H$ such that $C=\bigcap_{i=1}^{N} F\left(T_{i}\right) \neq \varnothing$. Let $\mu \in\left(0,2 \eta / \kappa^{2}\right)$, let $x_{0} \in H,\left\{\lambda_{n}\right\}_{n=1}^{\infty} \subset$ $[0,1)$, and let $\left\{\alpha_{n}\right\}_{n=1}^{\infty} \subset(0,1)$ satisfying the conditions: $\sum_{n=1}^{\infty} \lambda_{n}<\infty$ and $\alpha \leq \alpha_{n} \leq \beta, n \geq 1$, for some $\alpha, \beta \in(0,1)$. Then the sequence $\left\{x_{n}\right\}_{n=1}^{\infty}$ defined by (1.11) converges weakly to a common fixed point of the mappings $\left\{T_{i}\right\}_{i=1}^{N}$.

The above Theorem 1.6 extends Theorem 1.1 from the implicit iteration process (1.6) to the implicit iteration scheme (1.11) with perturbed mapping.

Let $E$ be a real Banach space, and let $K$ be a nonempty convex subset of $E$. Recall that a mapping $F: K \rightarrow K$ is said to be $\delta$-strongly accretive if there exists a constant $\delta \in(0,1)$ such that

$$
\langle F x-F y, j(x-y)\rangle \geq \delta\|x-y\|^{2}
$$

for all $x, y \in K$ and all $j(x-y) \in J(x-y)$.

Proposition 1.7. Let $X$ be a real Banach space, and let $F: K \rightarrow K$ be a mapping:

(i) if $F$ is $\lambda$-strictly pseudocontractive, then $F$ is a Lipschitz mapping with constant $L=1+$ $1 / \lambda$.

(ii) if $F$ is both $\lambda$-strictly pseudocontractive and $\delta$-strongly accretive with $\lambda+\delta \geq 1$, then $I-F$ is nonexpansive.

Proof. It is easy to see that statement (i) immediately follows from the definition of strict pseudocontraction. Now utilizing the definitions of strict pseudocontraction and strong accretivity, we obtain

$$
\lambda\|(I-F) x-(I-F) y\|^{2} \leq\|x-y\|^{2}-\langle F x-F y, j(x-y)\rangle \leq(1-\delta)\|x-y\|^{2} .
$$

Since $\lambda+\delta \geq 1$

$$
\|(I-F) x-(I-F) y\| \leq \sqrt{\frac{1-\delta}{\lambda}}\|x-y\| \leq\|x-y\|,
$$

and hence $I-F$ is nonexpansive.

Let $E$ be a real Banach space, and let $K$ be a nonempty convex subset of $E$ such that $K-K \subset K$. Let $\left\{T_{i}\right\}_{i=1}^{N}$ be $N$ strictly pseudocontractive self-maps of $K$, and let $F: K \rightarrow K$ be a perturbed mapping which is both $\delta$-strongly accretive and $\lambda$-strictly pseudocontractive with $\delta+\lambda \geq 1$. In this paper we introduce a general implicit iteration process as follows:

$$
\begin{aligned}
& x_{n}=\alpha_{n} x_{n-1}+\left(1-\alpha_{n}\right)\left[T_{n} y_{n}-\lambda_{n} F\left(T_{n} y_{n}\right)\right], \\
& y_{n}=\beta_{n} x_{n-1}+\left(1-\beta_{n}\right) T_{n} x_{n}, \quad n=1,2, \ldots
\end{aligned}
$$

where $T_{n}=T_{n \bmod N}$, and $\left\{\alpha_{n}\right\},\left\{\beta_{n}\right\},\left\{\lambda_{n}\right\} \subset[0,1]$. In particular, whenever $\lambda_{n} \equiv 0$, it is easy to see that (1.15) reduces to (1.8). 
Let $L \geq 1$ denote common Lipschitz constant of $N$ strictly pseudocontractive self-maps $\left\{T_{i}\right\}_{i=1}^{N}$ of $K$. Since $K$ is a nonempty convex subset of $E$ such that $K-K \subset K$, for each $n \geq 1$, the operator

$$
\begin{aligned}
S_{n} x & =\alpha_{n} x_{n-1}+\left(1-\alpha_{n}\right)\left\{T_{n}\left[\beta_{n} x_{n-1}+\left(1-\beta_{n}\right) T_{n} x\right]-\lambda_{n} F T_{n}\left[\beta_{n} x_{n-1}+\left(1-\beta_{n}\right) T_{n} x\right]\right\} \\
& =\alpha_{n} x_{n-1}+\left(1-\alpha_{n}\right)\left(I-\lambda_{n} F\right) T_{n}\left[\beta_{n} x_{n-1}+\left(1-\beta_{n}\right) T_{n} x\right] \\
& =\alpha_{n} x_{n-1}+\left(1-\alpha_{n}\right)\left[\left(1-\lambda_{n}\right) I+\lambda_{n}(I-F)\right] T_{n}\left[\beta_{n} x_{n-1}+\left(1-\beta_{n}\right) T_{n} x\right]
\end{aligned}
$$

maps $K$ into itself.

Utilizing Proposition 1.7, we have

$$
\begin{aligned}
\left\langle S_{n} x-\right. & \left.S_{n} y, j(x-y)\right\rangle \\
= & \left(1-\alpha_{n}\right)\left\langle\left[\left(1-\lambda_{n}\right) I+\lambda_{n}(I-F)\right] T_{n}\left[\beta_{n} x_{n-1}+\left(1-\beta_{n}\right) T_{n} x\right]\right. \\
& \left.\quad-\left[\left(1-\lambda_{n}\right) I+\lambda_{n}(I-F)\right] T_{n}\left[\beta_{n} x_{n-1}+\left(1-\beta_{n}\right) T_{n} y\right], j(x-y)\right\rangle \\
\leq & \left(1-\alpha_{n}\right) \|\left[\left(1-\lambda_{n}\right) I+\lambda_{n}(I-F)\right] T_{n}\left[\beta_{n} x_{n-1}+\left(1-\beta_{n}\right) T_{n} x\right] \\
& \quad-\left[\left(1-\lambda_{n}\right) I+\lambda_{n}(I-F)\right] T_{n}\left[\beta_{n} x_{n-1}+\left(1-\beta_{n}\right) T_{n} y\right]\|\| x-y \| \\
\leq & \left(1-\alpha_{n}\right)\left\{\left(1-\lambda_{n}\right)\left\|T_{n}\left[\beta_{n} x_{n-1}+\left(1-\beta_{n}\right) T_{n} x\right]-T_{n}\left[\beta_{n} x_{n-1}+\left(1-\beta_{n}\right) T_{n} y\right]\right\|\right. \\
& \left.\quad+\lambda_{n}\left\|(I-F) T_{n}\left[\beta_{n} x_{n-1}+\left(1-\beta_{n}\right) T_{n} x\right]-(I-F) T_{n}\left[\beta_{n} x_{n-1}+\left(1-\beta_{n}\right) T_{n} y\right]\right\|\right\} \\
& \times\|x-y\| \quad\left(1-\alpha_{n}\right)\left\|T_{n}\left[\beta_{n} x_{n-1}+\left(1-\beta_{n}\right) T_{n} x\right]-T_{n}\left[\beta_{n} x_{n-1}+\left(1-\beta_{n}\right) T_{n} y\right]\right\|\|x-y\| \\
\leq & \left(1-\alpha_{n}\right) L\left\|\beta_{n} x_{n-1}+\left(1-\beta_{n}\right) T_{n} x-\left[\beta_{n} x_{n-1}+\left(1-\beta_{n}\right) T_{n} y\right]\right\|\|x-y\| \\
= & \left(1-\alpha_{n}\right)\left(1-\beta_{n}\right) L\left\|T_{n} x-T_{n} y\right\|\|x-y\| \\
\leq & \left(1-\alpha_{n}\right)\left(1-\beta_{n}\right) L^{2}\|x-y\|^{2}
\end{aligned}
$$

for all $x, y \in K$. Thus, $S_{n}$ is strongly pseudocontractive, if $\left(1-\alpha_{n}\right)\left(1-\beta_{n}\right) L^{2}<1$ for each $n \geq 1$. Since $S_{n}$ is also Lipschitz mapping, it follows from $[12,15,16]$ that $S_{n}$ has a unique fixed point $x_{n} \in K$, that is, for each $n \geq 1$

$$
x_{n}=\alpha_{n} x_{n-1}+\left(1-\alpha_{n}\right)\left[\left(1-\lambda_{n}\right) I+\lambda_{n}(I-F)\right] T_{n}\left[\beta_{n} x_{n-1}+\left(1-\beta_{n}\right) T_{n} x_{n}\right] .
$$

Therefore, if $\left(1-\alpha_{n}\right)\left(1-\beta_{n}\right) L^{2}<1, \forall n \geq 1$, then the composite implicit iteration process (1.15) with perturbed mapping can be employed for the approximation of common fixed points of $N$ strictly pseudocontractive self-maps of $K$.

The purpose of this paper is to investigate the problem of approximating common fixed points of strictly pseudocontractive mappings of Browder-Petryshyn in an arbitrary real Banach space by this general implicit iteration process (1.15). To this end, we need the following lemma and definition. 
Lemma 1.8 (see [8]). Let $\left\{a_{n}\right\}_{n=1}^{\infty},\left\{b_{n}\right\}_{n=1}^{\infty}$, and $\left\{\epsilon_{n}\right\}_{n=1}^{\infty}$ be sequences of nonnegative real numbers satisfying the inequality

$$
a_{n+1} \leq\left(1+\epsilon_{n}\right) a_{n}+b_{n}, \quad n \geq 1 .
$$

If

$$
\sum_{n=1}^{\infty} \epsilon_{n}<+\infty, \quad \sum_{n=1}^{\infty} b_{n}<+\infty
$$

then $\lim _{n \rightarrow \infty} a_{n}$ exists.

The following definition can be found, for example, in [13].

Definition 1.9. Let $D$ be a closed subset of a real Banach space $E$, and let $T: D \rightarrow D$ be a mapping. $T$ is said to be semicompact if, for any bounded sequence $\left\{x_{n}\right\}$ in $D$ such that $\left\|x_{n}-T x_{n}\right\| \rightarrow 0(n \rightarrow \infty)$, there must exist a subsequence $\left\{x_{n_{i}}\right\} \subset\left\{x_{n}\right\}$ such that $x_{n_{i}} \rightarrow x^{*} \in$ D.

\section{Main Results}

We are now in a position to prove our main results in this paper.

Theorem 2.1. Let $E$ be a real Banach space, and let $K$ be a nonempty closed convex subset of $E$ such that $K-K \subset K$. Let $F: K \rightarrow K$ be a perturbed mapping which is both $\delta$-strongly accretive and $\lambda$-strictly pseudocontractive with $\delta+\lambda \geq 1$. Let $\left\{T_{i}\right\}_{i=1}^{N}$ be $N$ strictly pseudocontractive self-maps of $K$ such that $C=\bigcap_{i=1}^{N} F\left(T_{i}\right) \neq \varnothing$, where $F\left(T_{i}\right)=\left\{x \in K: T_{i} x=x\right\}$, and let $\left\{\alpha_{n}\right\}_{n=1}^{\infty},\left\{\beta_{n}\right\}_{n=1}^{\infty}$, and $\left\{\lambda_{n}\right\}_{n=1}^{\infty}$ be three real sequences in $[0,1]$ satisfying the conditions:

(i) $\sum_{n=1}^{\infty}\left(1-\alpha_{n}\right)=+\infty$;

(ii) $\sum_{n=1}^{\infty}\left(1-\alpha_{n}\right)^{2}<+\infty$;

(iii) $\sum_{n=1}^{\infty}\left(1-\beta_{n}\right)<+\infty$;

(iv) $\sum_{n=1}^{\infty} \lambda_{n}\left(1-\alpha_{n}\right)<+\infty$;

(v) $\left(1-\alpha_{n}\right)\left(1-\beta_{n}\right) L^{2}<1, \forall n \geq 1$, where $L \geq 1$ is the common Lipschitz constant of $\left\{T_{i}\right\}_{i=1}^{N}$.

For $x_{0} \in K$, let $\left\{x_{n}\right\}_{n=1}^{\infty}$ be defined by

$$
\begin{aligned}
& x_{n}=\alpha_{n} x_{n-1}+\left(1-\alpha_{n}\right)\left[T_{n} y_{n}-\lambda_{n} F\left(T_{n} y_{n}\right)\right], \\
& y_{n}=\beta_{n} x_{n-1}+\left(1-\beta_{n}\right) T_{n} x_{n}, \quad n=1,2, \ldots,
\end{aligned}
$$

where $T_{n}=T_{n \bmod N}$, then

(i) $\lim _{n \rightarrow \infty}\left\|x_{n}-p\right\|$ exists for all $p \in C$;

(ii) $\liminf _{n \rightarrow \infty}\left\|x_{n}-T_{n} x_{n}\right\|=0$.

Proof. First, since each strictly pseudocontractive mapping is a Lipschitz mapping, there exists a constant $L \geq 1$ such that

$$
\left\|T_{i} x-T_{i} y\right\| \leq L\|x-y\|, \quad \forall x, y \in K, \forall i=1,2, \ldots, N .
$$


It is now well known (see, e.g., [15]) that

$$
\|x+y\|^{2} \leq\|x\|^{2}+2\langle y, j(x+y)\rangle
$$

for all $x, y \in E$ and all $j(x+y) \in J(x+y)$. Take $p \in C$ arbitrarily. Then it follows from (2.1) that

$$
\begin{aligned}
x_{n}-p= & \alpha_{n} x_{n-1}+\left(1-\alpha_{n}\right)\left[T_{n} y_{n}-\lambda_{n} F\left(T_{n} y_{n}\right)\right]-p \\
= & \alpha_{n}\left(x_{n-1}-p\right)+\left(1-\alpha_{n}\right)\left\{\left[T_{n} y_{n}-\lambda_{n} F\left(T_{n} y_{n}\right)\right]-p\right\} \\
= & \alpha_{n}\left(x_{n-1}-p\right)+\left(1-\alpha_{n}\right)\left\{\left[\left(1-\lambda_{n}\right) I+\lambda_{n}(I-F)\right] T_{n} y_{n}-p\right\} \\
= & \alpha_{n}\left(x_{n-1}-p\right)+\left(1-\alpha_{n}\right)\left\{\left(1-\lambda_{n}\right)\left(T_{n} y_{n}-p\right)+\lambda_{n}\left[(I-F) T_{n} y_{n}-p\right]\right\} \\
= & \alpha_{n}\left(x_{n-1}-p\right)+\left(1-\alpha_{n}\right)\left\{\left(1-\lambda_{n}\right)\left(T_{n} y_{n}-T_{n} p\right)+\lambda_{n}\left[(I-F) T_{n} y_{n}-(I-F) T_{n} p\right]\right. \\
& \left.\quad+\lambda_{n}\left[(I-F) T_{n} p-p\right]\right\} \\
= & \alpha_{n}\left(x_{n-1}-p\right)+\left(1-\alpha_{n}\right)\left\{\left(1-\lambda_{n}\right)\left(T_{n} y_{n}-T_{n} p\right)+\lambda_{n}\left[(I-F) T_{n} y_{n}-(I-F) T_{n} p\right]\right\} \\
& -\left(1-\alpha_{n}\right) \lambda_{n} F(p) .
\end{aligned}
$$

Utilizing (2.3), we obtain

$$
\begin{aligned}
\left\|x_{n}-p\right\|^{2} \leq & \alpha_{n}^{2}\left\|x_{n-1}-p\right\|^{2}+2\left(1-\alpha_{n}\right)\left(1-\lambda_{n}\right)\left\langle T_{n} y_{n}-T_{n} p, j\left(x_{n}-p\right)\right\rangle \\
& +2\left(1-\alpha_{n}\right) \lambda_{n}\left\langle(I-F) T_{n} y_{n}-(I-F) T_{n} p, j\left(x_{n}-p\right)\right\rangle \\
& -2\left(1-\alpha_{n}\right) \lambda_{n}\left\langle F(p), j\left(x_{n}-p\right)\right\rangle \\
\leq & \alpha_{n}^{2}\left\|x_{n-1}-p\right\|^{2}+2\left(1-\alpha_{n}\right)\left(1-\lambda_{n}\right) \\
& \times\left[\left\langle T_{n} y_{n}-T_{n} x_{n}, j\left(x_{n}-p\right)\right\rangle+\left\langle T_{n} x_{n}-T_{n} p, j\left(x_{n}-p\right)\right\rangle\right] \\
& +2\left(1-\alpha_{n}\right) \lambda_{n}\left[\left\langle(I-F) T_{n} y_{n}-(I-F) T_{n} x_{n}, j\left(x_{n}-p\right)\right\rangle\right. \\
& \left.\quad+\left\langle(I-F) T_{n} x_{n}-(I-F) T_{n} p, j\left(x_{n}-p\right)\right\rangle\right] \\
& -2\left(1-\alpha_{n}\right) \lambda_{n}\left\langle F(p), j\left(x_{n}-p\right)\right\rangle .
\end{aligned}
$$

Since each $T_{i}, i=1,2, \ldots, N$, is strictly pseudocontractive, there exists $\lambda \in(0,1)$ such that

$$
\left\langle T_{i} x-T_{i} y, j(x-y)\right\rangle \leq\|x-y\|^{2}-\lambda\left\|x-T_{i} x-\left(y-T_{i} y\right)\right\|^{2}, \quad \forall x, y \in K .
$$


Thus, utilizing Proposition 1.7(ii) we know from (2.5) that

$$
\begin{aligned}
\left\|x_{n}-p\right\|^{2} \leq & \alpha_{n}^{2}\left\|x_{n-1}-p\right\|^{2}+2\left(1-\alpha_{n}\right)\left(1-\lambda_{n}\right) \\
& \times\left[L\left\|y_{n}-x_{n}\right\|\left\|x_{n}-p\right\|+\left\|x_{n}-p\right\|^{2}-\lambda\left\|x_{n}-T_{n} x_{n}\right\|^{2}\right] \\
& +2\left(1-\alpha_{n}\right) \lambda_{n}\left[L\left\|y_{n}-x_{n}\right\|\left\|x_{n}-p\right\|+L\left\|x_{n}-p\right\|^{2}\right] \\
& -2\left(1-\alpha_{n}\right) \lambda_{n}\left\langle F(p), j\left(x_{n}-p\right)\right\rangle \\
\leq & \alpha_{n}^{2}\left\|x_{n-1}-p\right\|^{2}+2\left(1-\alpha_{n}\right) \\
& \times\left[L\left\|y_{n}-x_{n}\right\|\left\|x_{n}-p\right\|+\left\|x_{n}-p\right\|^{2}-\lambda\left\|x_{n}-T_{n} x_{n}\right\|^{2}\right] \\
& +2\left(1-\alpha_{n}\right) \lambda_{n} L\left\|x_{n}-p\right\|^{2}+2\left(1-\alpha_{n}\right) \lambda_{n}\|F(p)\|\left\|x_{n}-p\right\| \\
\leq & \alpha_{n}^{2}\left\|x_{n-1}-p\right\|^{2}+2\left(1-\alpha_{n}\right) \\
& \times\left[L\left\|y_{n}-x_{n}\right\|\left\|x_{n}-p\right\|+\left\|x_{n}-p\right\|^{2}-\lambda\left\|x_{n}-T_{n} x_{n}\right\|^{2}\right] \\
& +2\left(1-\alpha_{n}\right) \lambda_{n} L\left\|x_{n}-p\right\|^{2}+\left(1-\alpha_{n}\right) \lambda_{n}\left(\|F(p)\|^{2}+\left\|x_{n}-p\right\|^{2}\right) \\
\leq & \alpha_{n}^{2}\left\|x_{n-1}-p\right\|^{2}+2\left(1-\alpha_{n}\right) \\
& \times\left[L\left\|y_{n}-x_{n}\right\|\left\|x_{n}-p\right\|+\left\|x_{n}-p\right\|^{2}-\lambda\left\|x_{n}-T_{n} x_{n}\right\|^{2}\right] \\
& +3 L\left(1-\alpha_{n}\right) \lambda_{n}\left\|x_{n}-p\right\|^{2}+\left(1-\alpha_{n}\right) \lambda_{n}\|F(p)\|^{2} .
\end{aligned}
$$

From (2.1), we also have that

$$
\begin{aligned}
\| y_{n}- & x_{n} \| \\
& \leq \beta_{n}\left\|x_{n}-x_{n-1}\right\|+\left(1-\beta_{n}\right)\left\|x_{n}-T_{n} x_{n}\right\| \\
& \leq \beta_{n}\left(1-\alpha_{n}\right)\left\|T_{n} y_{n}-\lambda_{n} F\left(T_{n} y_{n}\right)-x_{n-1}\right\|+\left(1-\beta_{n}\right)\left\|x_{n}-T_{n} x_{n}\right\| \\
\left\|T_{n} y_{n}-\lambda_{n} F\left(T_{n} y_{n}\right)-x_{n-1}\right\| & \leq\left\|T_{n} y_{n}-\lambda_{n} F\left(T_{n} y_{n}\right)-p\right\|+\left\|x_{n-1}-p\right\| \\
& =\left\|\left(1-\lambda_{n}\right)\left(T_{n} y_{n}-p\right)+\lambda_{n}\left((I-F) T_{n} y_{n}-p\right)\right\|+\left\|x_{n-1}-p\right\| \\
& =\left\|\left(1-\lambda_{n}\right)\left(T_{n} y_{n}-p\right)+\lambda_{n}\left((I-F) T_{n} y_{n}-(I-F) p\right)-\lambda_{n} F(p)\right\|+\left\|x_{n-1}-p\right\| \\
& \leq\left(1-\lambda_{n}\right)\left\|T_{n} y_{n}-p\right\|+\lambda_{n}\left\|T_{n} y_{n}-p\right\|+\lambda_{n}\|F(p)\|+\left\|x_{n-1}-p\right\| \\
& =\left\|T_{n} y_{n}-p\right\|+\lambda_{n}\|F(p)\|+\left\|x_{n-1}-p\right\| \\
& \leq L\left\|y_{n}-p\right\|+\lambda_{n}\|F(p)\|+\left\|x_{n-1}-p\right\| \\
& \leq\left(L \beta_{n}+1\right)\left\|x_{n-1}-p\right\|+L^{2}\left(1-\beta_{n}\right)\left\|x_{n}-p\right\|+\lambda_{n}\|F(p)\| .
\end{aligned}
$$


Since $T_{i}$ is a Lipschitz mapping with constant $L$, we have

$$
\left\|x_{n}-T_{n} x_{n}\right\| \leq\left\|x_{n}-p\right\|+\left\|T_{n} x_{n}-p\right\| \leq(L+1)\left\|x_{n}-p\right\| .
$$

Substituting (2.8) and (2.9) into (2.7), we deduce that

$$
\begin{aligned}
\left\|x_{n}-p\right\|^{2} \leq & \alpha_{n}^{2}\left\|x_{n-1}-p\right\|^{2}+2\left(1-\alpha_{n}\right)^{2} L \beta_{n}\left(L \beta_{n}+1\right)\left\|x_{n-1}-p\right\|\left\|x_{n}-p\right\| \\
& +2\left(1-\alpha_{n}\right)^{2} L^{3} \beta_{n}\left(1-\beta_{n}\right)\left\|x_{n}-p\right\|^{2} \\
& +2\left(1-\alpha_{n}\right)\left(1-\beta_{n}\right) L(L+1)\left\|x_{n}-p\right\|^{2} \\
& +2\left(1-\alpha_{n}\right)\left\|x_{n}-p\right\|^{2}-2\left(1-\alpha_{n}\right) \lambda\left\|x_{n}-T_{n} x_{n}\right\|^{2} \\
& +2 L\left(1-\alpha_{n}\right)^{2} \lambda_{n} \beta_{n}\|F(p)\|\left\|x_{n}-p\right\| \\
& +3 L\left(1-\alpha_{n}\right) \lambda_{n}\left\|x_{n}-p\right\|^{2}+\left(1-\alpha_{n}\right) \lambda_{n}\|F(p)\|^{2} \\
\leq & \alpha_{n}^{2}\left\|x_{n-1}-p\right\|^{2}+2\left(1-\alpha_{n}\right)^{2} L \beta_{n}\left(L \beta_{n}+1\right)\left\|x_{n-1}-p\right\|\left\|x_{n}-p\right\| \\
& +2\left(1-\alpha_{n}\right)^{2} L^{3} \beta_{n}\left(1-\beta_{n}\right)\left\|x_{n}-p\right\|^{2} \\
& +2\left(1-\alpha_{n}\right)\left(1-\beta_{n}\right) L(L+1)\left\|x_{n}-p\right\|^{2} \\
& +2\left(1-\alpha_{n}\right)\left\|x_{n}-p\right\|^{2}-2\left(1-\alpha_{n}\right) \lambda\left\|x_{n}-T_{n} x_{n}\right\|^{2} \\
& +4 L\left(1-\alpha_{n}\right) \lambda_{n}\left\|x_{n}-p\right\|^{2}+2 L\left(1-\alpha_{n}\right) \lambda_{n}\|F(p)\|^{2},
\end{aligned}
$$

and hence

$$
\begin{aligned}
{\left[1-2\left(1-\alpha_{n}\right)^{2} L^{3} \beta_{n}\left(1-\beta_{n}\right)-2\left(1-\alpha_{n}\right)\left(1-\beta_{n}\right) L(L+1)-4 L\left(1-\alpha_{n}\right) \lambda_{n}-2\left(1-\alpha_{n}\right)\right]\left\|x_{n}-p\right\|^{2} } \\
\leq \quad \alpha_{n}^{2}\left\|x_{n-1}-p\right\|^{2}+2\left(1-\alpha_{n}\right)^{2} L \beta_{n}\left(L \beta_{n}+1\right)\left\|x_{n-1}-p\right\|\left\|x_{n}-p\right\| \\
\quad-2\left(1-\alpha_{n}\right) \lambda\left\|x_{n}-T_{n} x_{n}\right\|^{2}+2 L\left(1-\alpha_{n}\right) \lambda_{n}\|F(p)\|^{2} .
\end{aligned}
$$

Setting

$$
b_{n}=2\left(1-\alpha_{n}\right)^{2} L^{3} \beta_{n}\left(1-\beta_{n}\right)+2\left(1-\alpha_{n}\right)\left(1-\beta_{n}\right) L(L+1)+4 L\left(1-\alpha_{n}\right) \lambda_{n},
$$


we conclude from (2.11) that

$$
\begin{aligned}
\left\|x_{n}-p\right\|^{2} \leq & \frac{\alpha_{n}^{2}}{1-2\left(1-\alpha_{n}\right)-b_{n}}\left\|x_{n-1}-p\right\|^{2}+\frac{2\left(1-\alpha_{n}\right)^{2} L \beta_{n}\left(L \beta_{n}+1\right)}{1-2\left(1-\alpha_{n}\right)-b_{n}}\left\|x_{n-1}-p\right\|\left\|x_{n}-p\right\| \\
& -\frac{2\left(1-\alpha_{n}\right) \lambda}{1-2\left(1-\alpha_{n}\right)-b_{n}}\left\|x_{n}-T_{n} x_{n}\right\|^{2}+\frac{\left(1-\alpha_{n}\right) \lambda_{n}}{1-2\left(1-\alpha_{n}\right)-b_{n}} 2 L\|F(p)\|^{2} .
\end{aligned}
$$

Thus

$$
\begin{aligned}
\left\|x_{n}-p\right\|^{2} \leq & {\left[1+\frac{\left(1-\alpha_{n}\right)^{2}+b_{n}}{1-2\left(1-\alpha_{n}\right)-b_{n}}\right]\left\|x_{n-1}-p\right\|^{2}+\frac{2\left(1-\alpha_{n}\right)^{2} L \beta_{n}\left(L \beta_{n}+1\right)}{1-2\left(1-\alpha_{n}\right)-b_{n}}\left\|x_{n-1}-p\right\|\left\|x_{n}-p\right\| } \\
& -2\left(1-\alpha_{n}\right) \lambda\left\|x_{n}-T_{n} x_{n}\right\|^{2}+\frac{\left(1-\alpha_{n}\right) \lambda_{n}}{1-2\left(1-\alpha_{n}\right)-b_{n}} 2 L\|F(p)\|^{2} .
\end{aligned}
$$

Since

$$
1-2\left(1-\alpha_{n}\right)-b_{n}=1-\left(1-\alpha_{n}\right)\left[2+2\left(1-\alpha_{n}\right) L^{3} \beta_{n}\left(1-\beta_{n}\right)+2\left(1-\beta_{n}\right) L(L+1)+4 L \lambda_{n}\right]
$$

and $\left\{\alpha_{n}\right\}_{n=1}^{\infty},\left\{\beta_{n}\right\}_{n=1}^{\infty},\left\{\lambda_{n}\right\}_{n=1}^{\infty} \subset[0,1]$, we get

$$
\left[2+2\left(1-\alpha_{n}\right) L^{3} \beta_{n}\left(1-\beta_{n}\right)+2\left(1-\beta_{n}\right) L(L+1)+4 L \lambda_{n}\right] \leq 6 L+2 L^{3}+2 L(L+1) .
$$

Setting $M_{1}=6 L+2 L^{3}+2 L(L+1)$, it follows from condition (ii) that $\lim _{n \rightarrow \infty}\left(1-\alpha_{n}\right)=0$ and so there must exist a natural number $N_{1}$ such that for all $n \geq N_{1}$,

$$
\frac{1}{1-2\left(1-\alpha_{n}\right)-b_{n}}<2
$$

Therefore, it follows from (2.14) that

$$
\begin{aligned}
\left\|x_{n}-p\right\|^{2} \leq & {\left[1+2\left(\left(1-\alpha_{n}\right)^{2}+b_{n}\right)\right]\left\|x_{n-1}-p\right\|^{2} } \\
& +2\left[2\left(1-\alpha_{n}\right)^{2} L \beta_{n}\left(L \beta_{n}+1\right)\right]\left\|x_{n-1}-p\right\|\left\|x_{n}-p\right\| \\
& -2\left(1-\alpha_{n}\right) \lambda\left\|x_{n}-T_{n} x_{n}\right\|^{2}+4 L\|F(p)\|^{2}\left(1-\alpha_{n}\right) \lambda_{n} .
\end{aligned}
$$


In order to consider the second term on the right-hand side of (2.18), we will prove that $\left\{x_{n}\right\}$ is bounded. Indeed, utilizing (2.8) and (2.9) and simplifying these inequalities, we have

$$
\begin{aligned}
\| x_{n}- & p \|^{2} \\
= & \left\langle x_{n}-p, j\left(x_{n}-p\right)\right\rangle \\
= & \alpha_{n}\left\langle x_{n-1}-p, j\left(x_{n}-p\right)\right\rangle+\left(1-\alpha_{n}\right)\left\langle T_{n} y_{n}-\lambda_{n} F\left(T_{n} y_{n}\right)-p, j\left(x_{n}-p\right)\right\rangle \\
= & \alpha_{n}\left\langle x_{n-1}-p, j\left(x_{n}-p\right)\right\rangle+\left(1-\alpha_{n}\right)\left(1-\lambda_{n}\right) \\
& \times\left[\left\langle T_{n} y_{n}-T_{n} x_{n}, j\left(x_{n}-p\right)\right\rangle+\left\langle T_{n} x_{n}-T_{n} p, j\left(x_{n}-p\right)\right\rangle\right] \\
& +\left(1-\alpha_{n}\right) \lambda_{n}\left\langle(I-F) T_{n} y_{n}-(I-F) T_{n} p, j\left(x_{n}-p\right)\right\rangle-\left(1-\alpha_{n}\right) \lambda_{n}\left\langle F(p), j\left(x_{n}-p\right)\right\rangle \\
\leq & \alpha_{n}\left\|x_{n-1}-p\right\|\left\|x_{n}-p\right\|+\left(1-\alpha_{n}\right)\left(1-\lambda_{n}\right)\left[L\left\|y_{n}-x_{n}\right\|\left\|x_{n}-p\right\|+L\left\|x_{n}-p\right\|^{2}\right] \\
& +\left(1-\alpha_{n}\right) \lambda_{n}\left[L\left\|y_{n}-x_{n}\right\|\left\|x_{n}-p\right\|+L\left\|x_{n}-p\right\|^{2}\right]+\left(1-\alpha_{n}\right) \lambda_{n}\|F(p)\|\left\|x_{n}-p\right\| \\
\leq & \alpha_{n}\left\|x_{n-1}-p\right\|\left\|x_{n}-p\right\|+\left(1-\alpha_{n}\right)\left[L\left\|y_{n}-x_{n}\right\|\left\|x_{n}-p\right\|+L\left\|x_{n}-p\right\|^{2}\right] \\
& +\left(1-\alpha_{n}\right) \lambda_{n}\|F(p)\|\left\|x_{n}-p\right\| \\
\leq & {\left[\alpha_{n}+L\left(1-\alpha_{n}\right)^{2} \beta_{n}\left(L \beta_{n}+1\right)\right]\left\|x_{n-1}-p\right\|\left\|x_{n}-p\right\| } \\
& +\left[\left(1-\alpha_{n}\right) L+L^{3}\left(1-\alpha_{n}\right)^{2} \beta_{n}\left(1-\beta_{n}\right)+L\left(1-\alpha_{n}\right)\left(1-\beta_{n}\right)(L+1)\right]\left\|x_{n}-p\right\|^{2} \\
& +(L+1)\left(1-\alpha_{n}\right) \lambda_{n}\|F(p)\|\left\|x_{n}-p\right\|, \\
& +L \beta_{n}\left(1-\alpha_{n}\right)^{2} \lambda_{n}\|F(p)\|\left\|x_{n}-p\right\|+\left(1-\alpha_{n}\right) \lambda_{n}\|F(p)\|\left\|x_{n}-p\right\| \\
& {\left[\alpha_{n}+L\left(1-\alpha_{n}\right)^{2} \beta_{n}\left(L \beta_{n}+1\right)\right]\left\|x_{n-1}-p\right\|\left\|x_{n}-p\right\| } \\
& {\left[\left(1-\alpha_{n}\right) L+L^{3}\left(1-\alpha_{n}\right)^{2} \beta_{n}\left(1-\beta_{n}\right)+L\left(1-\alpha_{n}\right)\left(1-\beta_{n}\right)(L+1)\right]\left\|x_{n}-p\right\|^{2} } \\
& +(1-p)
\end{aligned}
$$

and hence

$$
\begin{aligned}
& {\left[1-\left(1-\alpha_{n}\right) L-L^{3}\left(1-\alpha_{n}\right)^{2} \beta_{n}\left(1-\beta_{n}\right)-L\left(1-\alpha_{n}\right)\left(1-\beta_{n}\right)(L+1)\right]\left\|x_{n}-p\right\|^{2}} \\
& \leq\left[\alpha_{n}+L\left(1-\alpha_{n}\right)^{2} \beta_{n}\left(L \beta_{n}+1\right)\right]\left\|x_{n-1}-p\right\|\left\|x_{n}-p\right\| \\
& \quad+(L+1)\left(1-\alpha_{n}\right) \lambda_{n}\|F(p)\|\left\|x_{n}-p\right\| .
\end{aligned}
$$


This implies that

$$
\begin{aligned}
\left\|x_{n}-p\right\| & \\
\leq & \frac{\alpha_{n}+L\left(1-\alpha_{n}\right)^{2} \beta_{n}\left(L \beta_{n}+1\right)}{1-\left(1-\alpha_{n}\right) L-L^{3}\left(1-\alpha_{n}\right)^{2} \beta_{n}\left(1-\beta_{n}\right)-L\left(1-\alpha_{n}\right)\left(1-\beta_{n}\right)(L+1)}\left\|x_{n-1}-p\right\| \\
& +\frac{\left(1-\alpha_{n}\right) \lambda_{n}}{1-\left(1-\alpha_{n}\right) L-L^{3}\left(1-\alpha_{n}\right)^{2} \beta_{n}\left(1-\beta_{n}\right)-L\left(1-\alpha_{n}\right)\left(1-\beta_{n}\right)(L+1)}(L+1)\|F(p)\| \\
\leq & {\left[1+\frac{L^{3}\left(1-\alpha_{n}\right)^{2} \beta_{n}\left(1-\beta_{n}\right)+L\left(1-\alpha_{n}\right)\left(1-\beta_{n}\right)(L+1)+L\left(1-\alpha_{n}\right)^{2} \beta_{n}\left(L \beta_{n}+1\right)}{1-\left(1-\alpha_{n}\right) L-L^{3}\left(1-\alpha_{n}\right)^{2} \beta_{n}\left(1-\beta_{n}\right)-L\left(1-\alpha_{n}\right)\left(1-\beta_{n}\right)(L+1)}\right]\left\|x_{n-1}-p\right\| } \\
& +\frac{\left(1-\alpha_{n}\right) \lambda_{n}}{1-\left(1-\alpha_{n}\right) L-L^{3}\left(1-\alpha_{n}\right)^{2} \beta_{n}\left(1-\beta_{n}\right)-L\left(1-\alpha_{n}\right)\left(1-\beta_{n}\right)(L+1)}(L+1)\|F(p)\| .
\end{aligned}
$$

Now, we consider the second term on the right-hand side of (2.21). Since $\left\{\alpha_{n}\right\},\left\{\beta_{n}\right\} \subset[0,1]$, we have

$$
\left(1-\alpha_{n}\right)\left[L+L^{3}\left(1-\alpha_{n}\right) \beta_{n}\left(1-\beta_{n}\right)+L\left(1-\beta_{n}\right)(L+1)\right] \leq\left(1-\alpha_{n}\right)\left[L+L^{3}+L(L+1)\right] .
$$

Since $\lim _{n \rightarrow \infty}\left(1-\alpha_{n}\right)=0$, there exists a natural number $N_{2}\left(\geq N_{1}\right)$ such that for all $n \geq N_{2}$,

$$
1-\left(1-\alpha_{n}\right) L-L^{3}\left(1-\alpha_{n}\right)^{2} \beta_{n}\left(1-\beta_{n}\right)-L\left(1-\alpha_{n}\right)\left(1-\beta_{n}\right)(L+1) \geq \frac{1}{2} .
$$

Again, it follows from condition $\left\{\alpha_{n}\right\},\left\{\beta_{n}\right\} \subset[0,1]$ that

$$
\begin{aligned}
& L^{3}\left(1-\alpha_{n}\right)^{2} \beta_{n}\left(1-\beta_{n}\right)+L\left(1-\alpha_{n}\right)\left(1-\beta_{n}\right)(L+1)+L\left(1-\alpha_{n}\right)^{2} \beta_{n}\left(L \beta_{n}+1\right) \\
& \quad \leq L^{3}\left(1-\alpha_{n}\right)^{2}+L\left(1-\beta_{n}\right)(L+1)+L\left(1-\alpha_{n}\right)^{2}(L+1) .
\end{aligned}
$$

Therefore, it follows from (2.21) that

$$
\begin{aligned}
\left\|x_{n}-p\right\| \leq & \left\{1+2\left[L^{3}\left(1-\alpha_{n}\right)^{2}+L\left(1-\beta_{n}\right)(L+1)+L\left(1-\alpha_{n}\right)^{2}(L+1)\right]\right\}\left\|x_{n-1}-p\right\| \\
& +2\left(1-\alpha_{n}\right) \lambda_{n}(L+1)\|F(p)\| .
\end{aligned}
$$

According to conditions (ii)-(iv), we can readily see that

$$
\begin{gathered}
\sum_{n=1}^{\infty}\left\{2\left[L^{3}\left(1-\alpha_{n}\right)^{2}+L\left(1-\beta_{n}\right)(L+1)+L\left(1-\alpha_{n}\right)^{2}(L+1)\right]\right\}<+\infty \\
\sum_{n=1}^{\infty}\left\{2\left(1-\alpha_{n}\right) \lambda_{n}(L+1)\|F(p)\|\right\}<+\infty
\end{gathered}
$$


Thus, in terms of Lemma 1.8 we deduce that $\lim _{n \rightarrow \infty}\left\|x_{n}-p\right\|$ exists, and hence $\left\{x_{n}\right\}$ is bounded.

Now, we consider the second term on the right-hand side of (2.18). Since $\left\{x_{n}\right\}$ is bounded, and $\left\{\beta_{n}\right\}_{n=1}^{\infty} \subset[0,1]$, there exists a constant $M_{2}>0$ and a natural number $N_{3}\left(\geq N_{2}\right)$ such that for all $n \geq N_{3}$,

$$
2\left[2\left(1-\alpha_{n}\right)^{2} L \beta_{n}\left(L \beta_{n}+1\right)\right]\left\|x_{n-1}-p\right\|\left\|x_{n}-p\right\| \leq 2\left(1-\alpha_{n}\right)^{2} M_{2} .
$$

Thus, it follows from (2.18) that

$$
\begin{aligned}
\left\|x_{n}-p\right\|^{2} \leq & {\left[1+2\left(\left(1-\alpha_{n}\right)^{2}+b_{n}\right)\right]\left\|x_{n-1}-p\right\|^{2}+2\left(1-\alpha_{n}\right)^{2} M_{2} } \\
& -2\left(1-\alpha_{n}\right) \lambda\left\|x_{n}-T_{n} x_{n}\right\|^{2}+4 L\|F(p)\|^{2}\left(1-\alpha_{n}\right) \lambda_{n} .
\end{aligned}
$$

Since $\left\{x_{n}\right\}$ is bounded, there exists a constant $M_{3}>0$ such that $\left\|x_{n}-p\right\|^{2} \leq M_{3}$. It follows from (2.28) that

$$
\begin{aligned}
2 \lambda \sum_{j=N+1}^{n}\left(1-\alpha_{j}\right)\left\|x_{j}-T_{j} x_{j}\right\|^{2} \leq & \left\|x_{N}-p\right\|^{2}+M_{3} \sum_{j=N+1}^{n} 2\left[\left(1-\alpha_{j}\right)^{2}+b_{j}\right] \\
& +2 M_{2} \sum_{j=N+1}^{n}\left(1-\alpha_{j}\right)^{2}+4 L\|F(p)\|^{2} \sum_{j=N+1}^{n}\left(1-\alpha_{j}\right) \lambda_{j},
\end{aligned}
$$

and hence

$$
\begin{aligned}
2 \lambda \sum_{n=N+1}^{\infty}\left(1-\alpha_{n}\right)\left\|x_{n}-T_{n} x_{n}\right\|^{2} \leq & \left\|x_{N}-p\right\|^{2}+M_{3} \sum_{n=N+1}^{\infty} 2\left[\left(1-\alpha_{n}\right)^{2}+b_{n}\right] \\
& +2 M_{2} \sum_{n=N+1}^{\infty}\left(1-\alpha_{n}\right)^{2}+4 L\|F(p)\|^{2} \sum_{n=N+1}^{\infty}\left(1-\alpha_{n}\right) \lambda_{n} .
\end{aligned}
$$

Utilizing conditions (ii)-(iv), we know from (2.30) that

$$
2 \lambda \sum_{n=1}^{\infty}\left(1-\alpha_{n}\right)\left\|x_{n}-T_{n} x_{n}\right\|^{2}<+\infty
$$

Since $\sum_{n=1}^{\infty}\left(1-\alpha_{n}\right)=+\infty$, we have

$$
\liminf _{n \rightarrow \infty}\left\|x_{n}-T_{n} x_{n}\right\|=0
$$

This completes the proof of Theorem 2.1. 
Fixed Point Theory and Applications

The iterative scheme (1.15) becomes the explicit version as follows, whenever $\beta_{n} \equiv 1$ :

$$
x_{n}=\alpha_{n} x_{n-1}+\left(1-\alpha_{n}\right)\left[T_{n} x_{n-1}-\lambda_{n} F\left(T_{n} x_{n-1}\right)\right], \quad n \geq 1 .
$$

In the case when $N=1,(2.33)$ is the Mann iteration process as follows:

$$
x_{n}=\alpha_{n} x_{n-1}+\left(1-\alpha_{n}\right)\left[T x_{n-1}-\lambda_{n} F\left(T x_{n-1}\right)\right], \quad n \geq 1 .
$$

The conclusion of Theorem 2.1 remains valid for the iteration processes (2.33) and (2.34). Furthermore, we have the following result.

Theorem 2.2. Let $E$ be a real Banach space, and let $K$ be a nonempty closed convex subset of $E$ such that $K-K \subset K$. Let $F: K \rightarrow K$ be a perturbed mapping which is both $\delta$-strongly accretive and $\lambda$ strictly pseudocontractive with $\delta+\lambda \geq 1$. Let $T$ be a semicompact strictly pseudocontractive self-map of $K$ such that $F(T) \neq \varnothing$, where $F(T)=\{x \in K: T x=x\}$, and let $\left\{\alpha_{n}\right\}_{n=1}^{\infty}$ and $\left\{\lambda_{n}\right\}_{n=1}^{\infty}$ be two real sequences in $[0,1]$ satisfying the conditions:

(i) $\sum_{n=1}^{\infty}\left(1-\alpha_{n}\right)=+\infty$;

(ii) $\sum_{n=1}^{\infty}\left(1-\alpha_{n}\right)^{2}<+\infty$;

(iii) $\sum_{n=1}^{\infty} \lambda_{n}\left(1-\alpha_{n}\right)<+\infty$.

Then Mann iteration process (2.34) converges strongly to a fixed point of T.

Proof. Since

$$
\liminf _{n \rightarrow \infty}\left\|x_{n}-T x_{n}\right\|=0,
$$

there exists a subsequence $\left\{n_{k}\right\}$ of $\{n\}$ such that

$$
\lim _{k \rightarrow \infty}\left\|x_{n_{k}}-T x_{n_{k}}\right\|=0
$$

By the semicompactness of $T$, there must exist a subsequence $\left\{x_{n_{k_{i}}}\right\}$ of $\left\{x_{n_{k}}\right\}$ such that

$$
\lim _{i \rightarrow \infty} x_{n_{k_{i}}}=p_{0}
$$

It follows from (2.36) that $p_{0}=T p_{0}$, and hence $p_{0} \in F(T)$. Since $\lim _{n \rightarrow \infty}\left\|x_{n}-p_{0}\right\|$ exists, we have

$$
\lim _{n \rightarrow \infty}\left\|x_{n}-p_{0}\right\|=\lim _{i \rightarrow \infty}\left\|x_{n_{k_{i}}}-p_{0}\right\|=0
$$

This completes the proof of Theorem 2.2. 


\section{Acknowledgments}

The first author was partially supported by the National Science Foundation of China (10771141), Ph.D. Program Foundation of Ministry of Education of China (20070270004), and Science and Technology Commission of Shanghai Municipality grant (075105118), Leading Academic Discipline Project of Shanghai Normal University (DZL707), Shanghai Leading Academic Discipline Project (S30405) and Innovation Program of Shanghai Municipal Education Commission (09ZZ133). The third author was partially supported by the Grant NSF 97-2115-M-110-001

\section{References}

[1] F. E. Browder and W. V. Petryshyn, "Construction of fixed points of nonlinear mappings in Hilbert space," Journal of Mathematical Analysis and Applications, vol. 20, pp. 197-228, 1967.

[2] T. L. Hicks and J. D. Kubicek, "On the Mann iteration process in a Hilbert space," Journal of Mathematical Analysis and Applications, vol. 59, no. 3, pp. 498-504, 1977.

[3] S.. Măruşter, "The solution by iteration of nonlinear equations in Hilbert spaces," Proceedings of the American Mathematical Society, vol. 63, no. 1, pp. 69-73, 1977.

[4] M. O. Osilike and A. Udomene, "Demiclosedness principle and convergence theorems for strictly pseudocontractive mappings of Browder-Petryshyn type," Journal of Mathematical Analysis and Applications, vol. 256, no. 2, pp. 431-445, 2001.

[5] M. O. Osilike, "Strong and weak convergence of the Ishikawa iteration method for a class of nonlinear equations," Bulletin of the Korean Mathematical Society, vol. 37, no. 1, pp. 153-169, 2000.

[6] B. E. Rhoades, "Comments on two fixed point iteration methods," Journal of Mathematical Analysis and Applications, vol. 56, no. 3, pp. 741-750, 1976.

[7] B. E. Rhoades, "Fixed point iterations using infinite matrices," Transactions of the American Mathematical Society, vol. 196, pp. 161-176, 1974.

[8] M. O. Osilike, S. C. Aniagbosor, and B. G. Akuchu, "Fixed points of asymptotically demicontractive mappings in arbitrary Banach spaces," PanAmerican Mathematical Journal, vol. 12, no. 2, pp. 77-88, 2002.

[9] L.-C. Zeng, G. M. Lee, and N. C. Wong, "Ishikawa iteration with errors for approximating fixed points of strictly pseudocontractive mappings of Browder-Petryshyn type," Taiwanese Journal of Mathematics, vol. 10, no. 1, pp. 87-99, 2006.

[10] L.-C. Zeng, N.-C. Wong, and J.-C. Yao, "Strong convergence theorems for strictly pseudocontractive mappings of Browder-Petryshyn type," Taiwanese Journal of Mathematics, vol. 10, no. 4, pp. 837-849, 2006.

[11] H.-K. Xu and R. G. Ori, "An implicit iteration process for nonexpansive mappings," Numerical Functional Analysis and Optimization, vol. 22, no. 5-6, pp. 767-773, 2001.

[12] M. O. Osilike, "Implicit iteration process for common fixed points of a finite family of strictly pseudocontractive maps," Journal of Mathematical Analysis and Applications, vol. 294, no. 1, pp. 73-81, 2004.

[13] Y. Su and S. Li, "Composite implicit iteration process for common fixed points of a finite family of strictly pseudocontractive maps," Journal of Mathematical Analysis and Applications, vol. 320, no. 2, pp. 882-891, 2006.

[14] L.-C. Zeng and J.-C. Yao, "Implicit iteration scheme with perturbed mapping for common fixed points of a finite family of nonexpansive mappings," Nonlinear Analysis: Theory, Methods $\mathcal{E}$ Applications, vol. 64, no. 11, pp. 2507-2515, 2006.

[15] S.-S. Chang, "Some problems and results in the study of nonlinear analysis," Nonlinear Analysis: Theory, Methods E Applications, vol. 30, no. 7, pp. 4197-4208, 1997.

[16] Z.-H. Sun, "Strong convergence of an implicit iteration process for a finite family of asymptotically quasi-nonexpansive mappings," Journal of Mathematical Analysis and Applications, vol. 286, no. 1, pp. 351-358, 2003. 